\title{
The Progenitor-Remnant Connection of Neutrino-Driven Supernovae Across the Stellar Mass Range
}

\author{
Thomas Ertl \\ Max-Planck-Institut für Astrophysik, \\ Postfach 1317, D-85741 Garching, Germany \\ email: tertl@mpa-garching.mpg.de
}

\begin{abstract}
We perform hydrodynamic supernova (SN) simulations in spherical symmetry for progenitor models with solar metallicity across the stellar mass range from 9.0 to $120 M_{\odot}$ to explore the progenitor-explosion and progenitor-remnant connections based on the neutrinodriven mechanism. We use an approximative treatment of neutrino transport and replace the high-density interior of the neutron star (NS) by an inner boundary condition based on an analytic proto-NS core-cooling model, whose free parameters are chosen to reproduce the observables of SN 1987A and the Crab SN for theoretical models of their progenitor stars.

Judging the fate of a massive star, either a neutron star (NS) or a black hole (BH), solely by its structure prior to collapse has been ambiguous. Our work and previous attempts find a non-monotonic variation of successful and failed supernovae with zero-age main-sequence mass. We identify two parameters based on the "critical luminosity" concept for neutrino-driven explosions, which in combination allows for a clear separation of exploding and non-exploding cases.

Continuing our simulations beyond shock break-out, we are able to determine nucleosynthesis, light curves, explosion energies, and remnant masses. The resulting NS initial mass function has a mean gravitational mass near $1.4 M_{\odot}$. The average $\mathrm{BH}$ mass is about $9 M_{\odot}$ if only the helium core implodes, and $14 M_{\odot}$ if the entire pre-SN star collapses. Only $\sim 10 \%$ of SNe come from stars over $20 M_{\odot}$, and some of these are Type Ib or Ic.
\end{abstract}

Keywords. nuclear reactions, nucleosynthesis, abundances, stars: black holes, stars: massive, stars: neutron, supernovae: general, hydrodynamics, neutrinos

\section{Introduction}

Recent observations in the past years have provided a picture of the population of core-collapse supernovae (CCSNe) in various aspects: Progenitor stars of Type-IIP SNe (Smartt, 2015) have been identified and suggested a lack of high-mass stars exploding as $\mathrm{SNe}$; mass measurements of black holes (BHs) and neutron stars (NSs) in binary systems have provided a sketch of the mass distributions (e.g., Özel \& Freire, 2016, and references therein); the elemental abundance patterns (for the sun see Lodders, 2003) pose a mixed imprint of all the sources of heavy elements including the contribution by CCSNe. These observational signatures connect progenitor stars with remnant and explosion properties.

Theoretical studies of CCSNe, however, have barely tapped these observational resources and a converged theoretical picture has not yet emerged, although considerable progress has been made in recent years with the first successful self-consistent explosions in full geometry (3D; Melson et al., 2015b;a, Lentz et al., 2015). However, computational power restricts evolving these simulations for sufficiently long time to gain converged observable signatures and a large number of models is also computationally unaffordable. Nevertheless, any successful explosion mechanism needs to reproduce the observed 


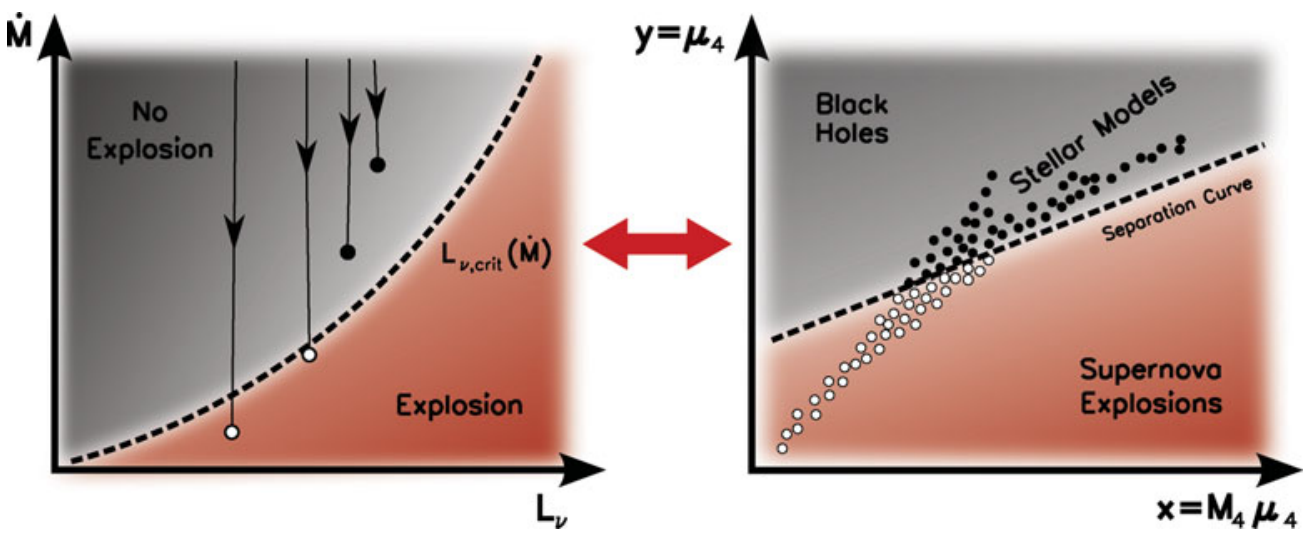

Figure 1. (Taken from Ertl et al., 2016, Fig. 6) Correspondence of $L_{\nu}-\dot{M}$ plane with critical neutrino luminosity $L_{\nu, \mathrm{crit}}(\dot{M})$ (left) and $x-y$ plane with separation curve (right). In the left plot post-bounce evolution paths of successfully exploding models (white circles) and non-exploding models (black circles) are schematically indicated, corresponding to white and black circles for pre-collapse models in the right plot. Evolution paths of successful models cross the critical line at some point and the accretion ends after the explosion has taken off. In contrast, the tracks of failing cases never reach the critical conditions for launching the runaway expansion of the shock. The symbols in the left plot mark the "optimal point" relative to the critical curve that can be reached, corresponding to the stellar conditions described by the parameters $\left(M_{4} \mu_{4}\right.$ and $\left.\mu_{4}\right)$ at the $s=4$ location, which seems decisive for the success or failure of the explosion of a progenitor, because the accretion rate drops strongly outside.

population of SNe and their remnants. The delayed neutrino-driven mechanism is the best candidate for a mechanism and certainly the most elaborate.

We investigated the properties of the progenitor stars and their SN explosions by a systematic parameter approach. The novel aspect of the approach is that the explosions are not triggered artificially by a piston or a thermal bomb, but are based on the current understanding of the neutrino-driven mechanism. We use an approximative neutrino transport solver and excise the inner core of the proto-NS and replace it by an analytic one-zone core-cooling model (Ugliano et al., 2012), whose free parameters are tuned to reproduce SN 1987A and the Crab SN for theoretical models of their progenitors. Both $\mathrm{SNe}$ act as our observational anchors of the survey. The obtained parameter choice is then applied to progenitor models of different zero-age main-sequence (ZAMS) masses and evaluated for the explosion properties.

\section{A Two-Parameter Criterion for the Explodability of Massive Stars}

In Ertl et al. (2016), we published a criterion for the "explodability" of massive stars, which is solely based on the pre-collapse structure of the star. Two parameters were identified based on the "critical luminosity concept" (Burrows \& Goshy, 1993). One is the normalized mass inside a dimensionless entropy per nucleon of $s=4$,

$$
M_{4} \equiv m(s=4) / M_{\odot},
$$

and the mass derivative at this location,

$$
\left.\mu_{4} \equiv \frac{\mathrm{d} m / M_{\odot}}{\mathrm{d} r / 1000 \mathrm{~km}}\right|_{s=4},
$$

which allow for a nearly perfect prediction of exploding and non-exploding cases in our study ( $\gtrsim 97 \%$ of all models are correctly predicted). Fig. 1 depicts the correspondence. 


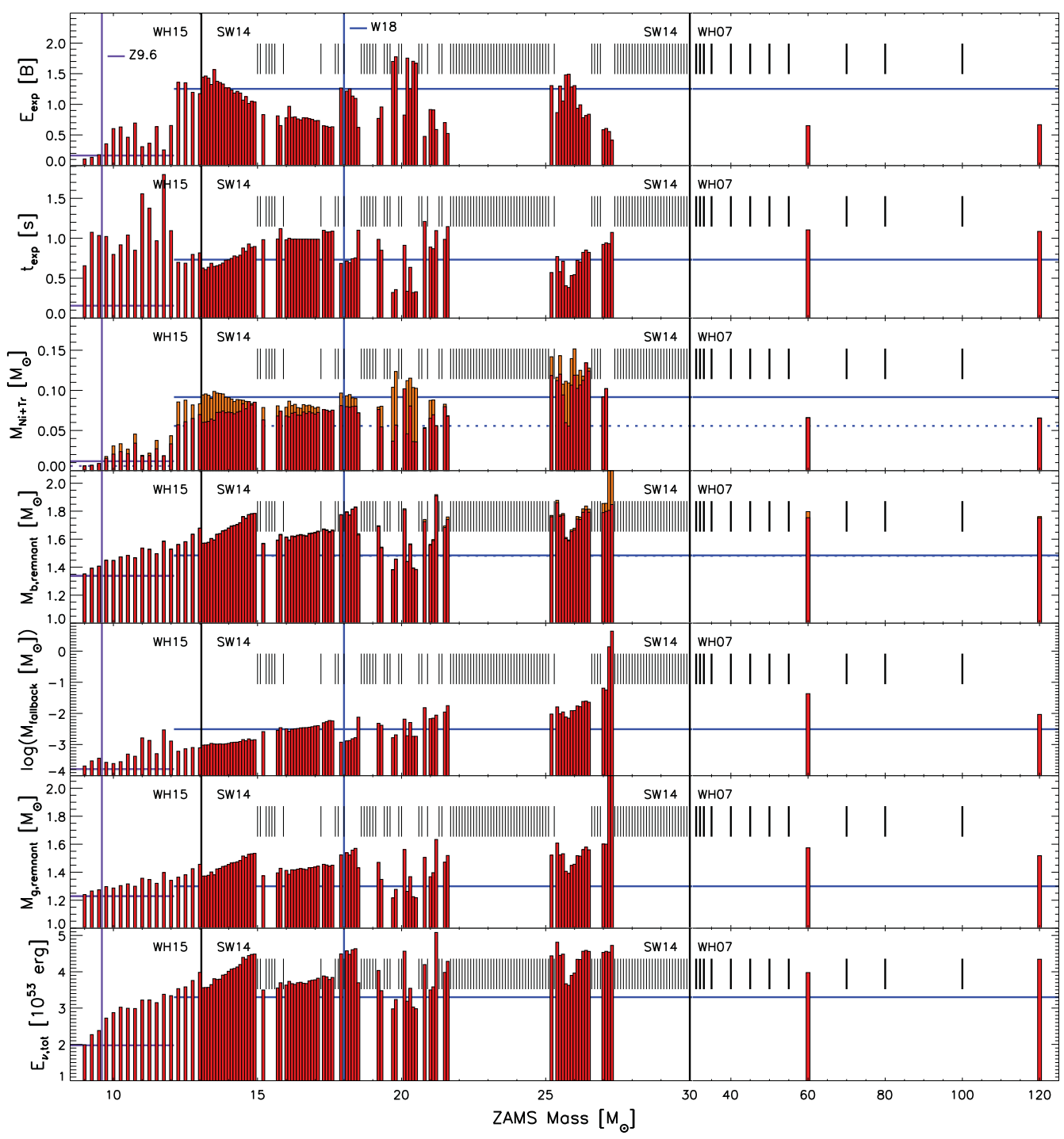

Figure 2. (Taken from Sukhbold et al., 2016, Fig. 8) Explosion properties for all models exploded with the Z9.6 and W18 calibrations. Black vertical lines mark the boundaries between the different progenitor sets our model sample is composed of. The panels, from top to bottom, show the final explosion energy, $\mathrm{E}$, in units of $1 \mathrm{~B}=1$ Bethe $=10^{51} \mathrm{erg}$, the time of the onset of the explosion, $t_{\exp }$, the mass of finally ejected, explosively produced ${ }^{56} \mathrm{Ni}$ (red bars) and tracer element environment (orange bars), the baryonic mass of the compact remnant with the fallback mass indicated by orange sections on the bars, the fallback mass, the gravitational mass of the compact remnant, and the total energy radiated in neutrinos, $E_{\nu, \text { tot }}$. The masses of the calibration models are indicated by vertical blue lines, and the corresponding results by horizontal solid or dashed blue lines, which extend over the mass ranges that are considered to have Crab-like or SN1987A-like progenitor properties, respectively. Non-exploding cases are marked by short vertical black bars in the upper half of each panel.

\section{Nucleosynthesis, light curves, explosion energies, and remnant masses}

Continuing the simulations beyond the point of shock break-out, we were able to determine explosion energies and remnant masses as well as nucleosynthesis yields and 
light curves in a post-processing step. The results for a dense set of progenitor models with solar metallicity are reported in Sukhbold et al. (2016). Fig. 2 shows the results of our study for one core-model parameter choice as an example. The parameters were calibrated employing the blue supergiant model "W18" (see Sukhbold et al., 2016, for details).

This work was supported by Deutsche Forschungsgemeinschaft through grant EXC 153 "Excellence Cluster Universe" and the European Research Council through grant ERC-AdG No. 341157-COCO2CASA.

\section{References}

Burrows, A. \& Goshy, J. 1993, ApJ (Letters), 416, L75

Ertl, T., Janka, H.-T., Woosley, S. E., Sukhbold, T., \& Ugliano, M. 2016, ApJ, 818, 124

Özel, F. \& Freire, P. 2016, ARA\&A A, 54, 401

Lentz, E. J., Bruenn, S. W., Hix, W. R., et al. 2015, ApJ (Letters), 807, L31

Lodders, K. 2003, ApJ, 591, 1220

Melson, T., Janka, H.-T., \& Marek, A. 2015, ApJ (Letters), 801, L24

Melson, T., Janka, H.-T., Bollig, R., et al. 2015, ApJ (Letters), 808, L42

Smartt, S. J. 2015, PASA, 32, e016

Sukhbold, T., Ertl, T., Woosley, S. E., Brown, J. M., \& Janka, H.-T. 2016, ApJ, 821, 38

Ugliano, M., Janka, H.-T., Marek, A., \& Arcones, A. 2012, ApJ, 757, 69 